\title{
Non - Photocatalytic Water Splitting Process to Generate Green Electricity in Alkali Doped Zinc Oxide based Hydroelectric Cell
}

\section{Rekha Gupta $^{1}$, Jyoti Shah ${ }^{1}$, Rakesh Singh ${ }^{2}$, R. K. Kotnala ${ }^{1 *}$}

${ }^{1}$ CSIR-National Physical Laboratory, Dr. K. S. Krishnan Road, New Delhi-110012, India

${ }^{2}$ University Center for Nanoscience and Nanotechnology

Aryabhatta Knowledge University Patna, Mithapur, Patna - 800001, India

*Corresponding Author

E-mail: rkkotnala@gmail.com,Fax: 91-11-45609310

S1. Microstrain $(\varepsilon)$ induced in the lattices of $\mathrm{ZnO}$ by alkali metal ion doping was calculated by Williamson-Hall equation : $\beta_{h k l} \cos \theta=\frac{K \lambda}{d}+4 \varepsilon \sin \theta$, where $\beta_{h k l}$ is instrument broadening, $\theta$ is angle of diffraction in radian, $\mathrm{K}$ is shape factor, $\mathrm{d}$ is crystallite size, $\lambda$ is wavelength of $\mathrm{CuK}_{\alpha}$ radiation and $\varepsilon$ is strain induced in unit cell of $\mathrm{ZnO}$.
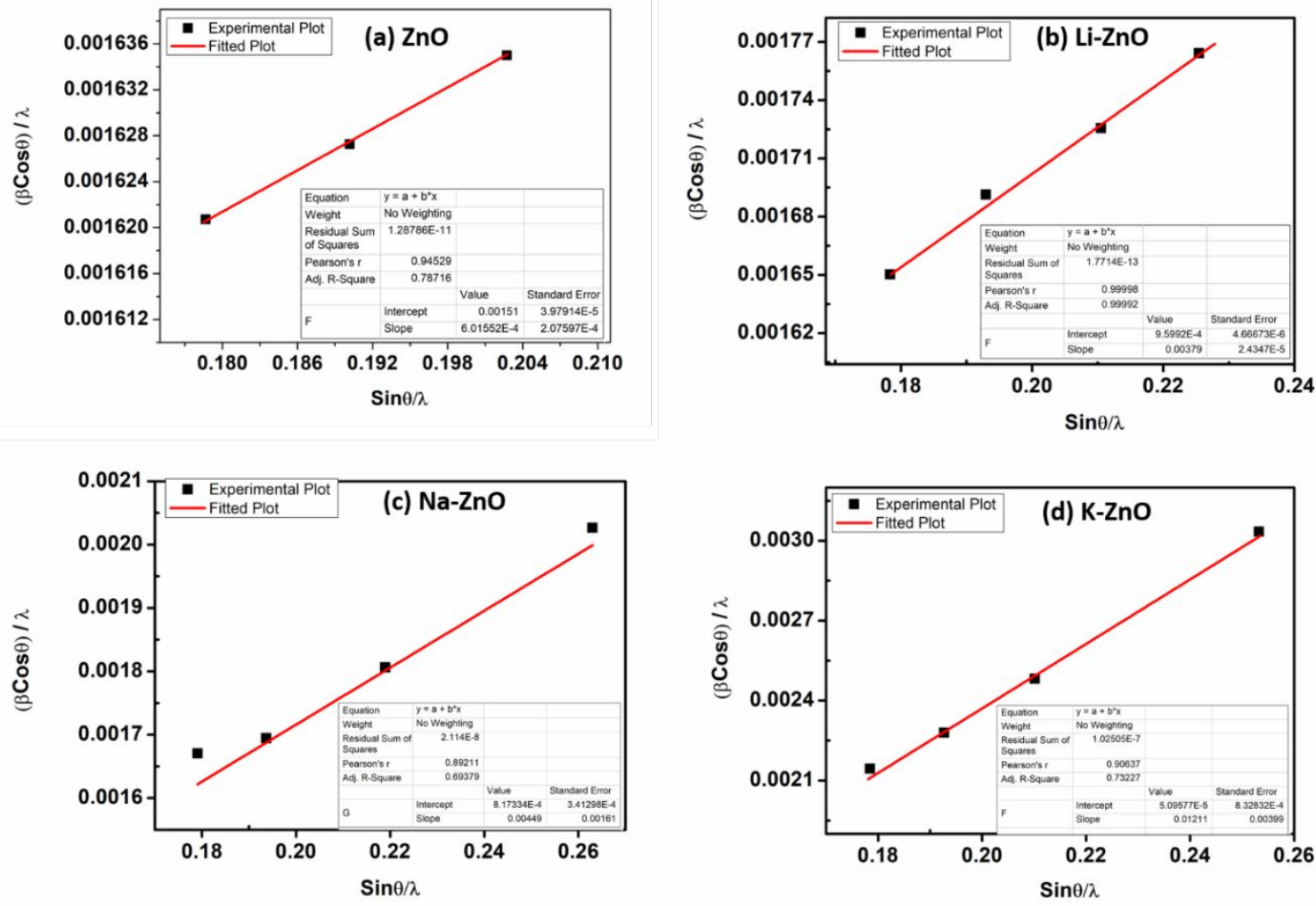

Fig. S1 Williamson's Hall plots of (a) Pure ZnO (b) Li-ZnO (c) Na-ZnO (d) K-ZnO 
Fig. S1 represents Williamson's-Hall plots of pure and alkali doped ZnO samples. The microstrain was calculated from slope of the plots given in Fig.S1

S2. EDAX analysis and TEM images of pure and alkali metal doped $\mathrm{ZnO}$ samples are represented in Fig.S2.

(1) Pure ZnO:

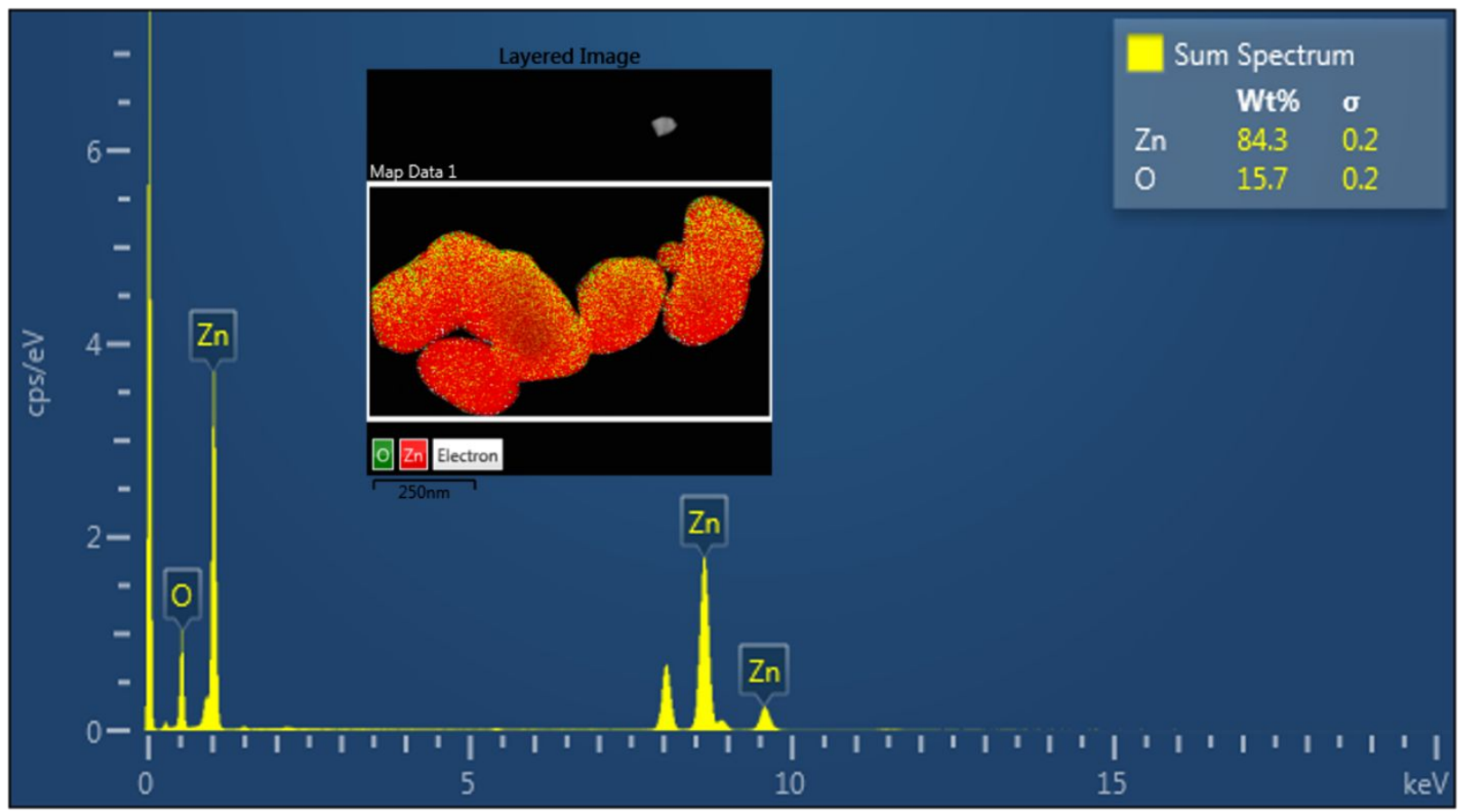

\begin{tabular}{|l|r|r|l|r|r|r|r|}
\hline Element & $\begin{array}{c}\text { Line } \\
\text { Type }\end{array}$ & k Factor & k Factor type & $\begin{array}{c}\text { Absorption } \\
\text { Correction }\end{array}$ & Wt\% & \multicolumn{1}{|c|}{$\begin{array}{c}\text { Wt\% } \\
\text { Sigma }\end{array}$} & Atomic \% \\
\hline $\mathrm{O}$ & K series & 1.455 & Theoretical & 1.00 & 15.67 & 0.18 & 43.15 \\
\hline $\mathrm{Zn}$ & K series & 1.496 & Theoretical & 1.00 & 84.33 & 0.18 & 56.85 \\
\hline Total: & & & & & 100.00 & & 100.00 \\
\hline
\end{tabular}

(2) Li doped $\mathrm{ZnO}$

Li could not be detected in EDAX spectra due to low sensitivity issues. However presence of Li was confirmed in Elemental Mapping shown in image below: 


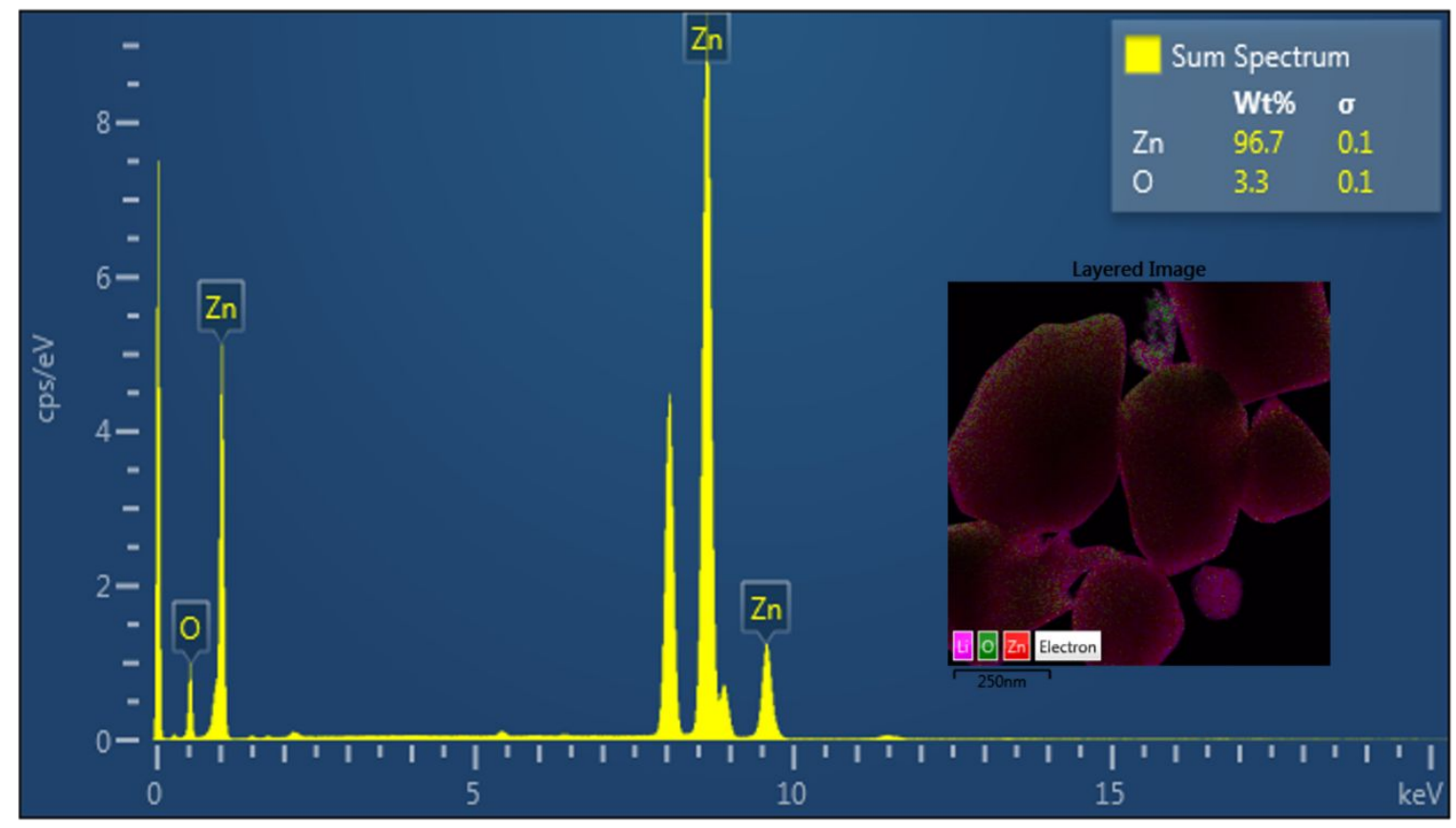

\begin{tabular}{|l|r|r|l|r|r|r|r|}
\hline Element & $\begin{array}{c}\text { Line } \\
\text { Type }\end{array}$ & k Factor & k Factor type & $\begin{array}{c}\text { Absorption } \\
\text { Correction }\end{array}$ & Wt\% & \multicolumn{1}{|c|}{$\begin{array}{c}\text { Wt\% } \\
\text { Sigma }\end{array}$} & Atomic \% \\
\hline $\mathrm{O}$ & K series & 1.455 & Theoretical & 1.00 & 3.32 & 0.05 & 12.32 \\
\hline $\mathrm{Zn}$ & K series & 1.496 & Theoretical & 1.00 & 96.68 & 0.05 & 87.68 \\
\hline Total: & & & & & 100.00 & & 100.00 \\
\hline
\end{tabular}

(3) Na doped ZnO

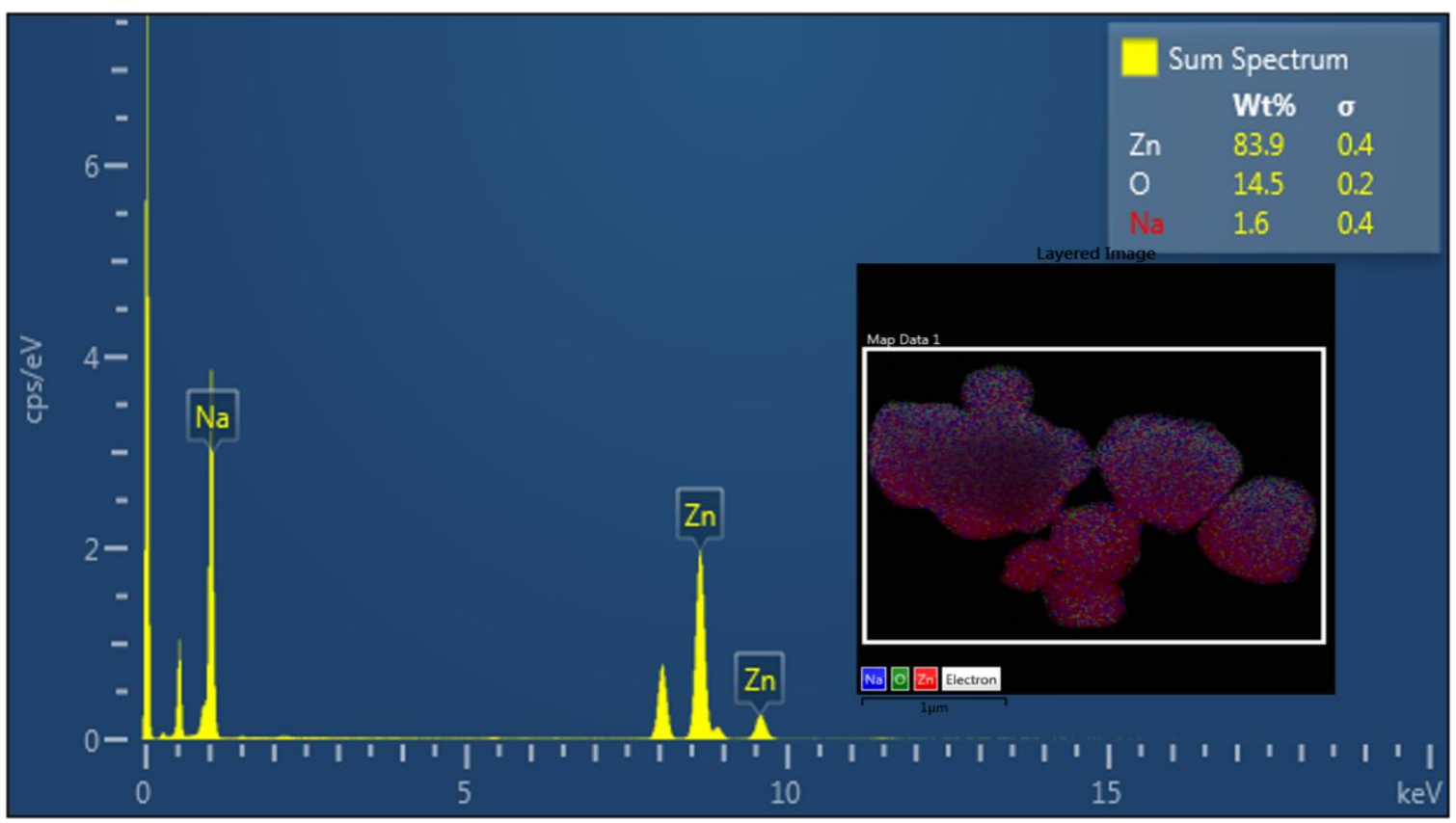




\begin{tabular}{|l|c|r|l|r|r|r|r|}
\hline Element & $\begin{array}{c}\text { Line } \\
\text { Type }\end{array}$ & k Factor & k Factor type & $\begin{array}{c}\text { Absorption } \\
\text { Correction }\end{array}$ & Wt\% & \multicolumn{1}{|c|}{$\begin{array}{c}\text { Wt\% } \\
\text { Sigma }\end{array}$} & Atomic \% \\
\hline $\mathrm{O}$ & K series & 1.455 & Theoretical & 1.00 & 14.46 & 0.21 & 40.03 \\
\hline $\mathrm{Na}$ & K series & 1.124 & Theoretical & 1.00 & 1.62 & 0.35 & 3.12 \\
\hline $\mathrm{Zn}$ & K series & 1.496 & Theoretical & 1.00 & 83.92 & 0.36 & 56.85 \\
\hline Total: & & & & & 100.00 & & 100.00 \\
\hline
\end{tabular}

(4) $\mathrm{K}$ doped $\mathrm{ZnO}$

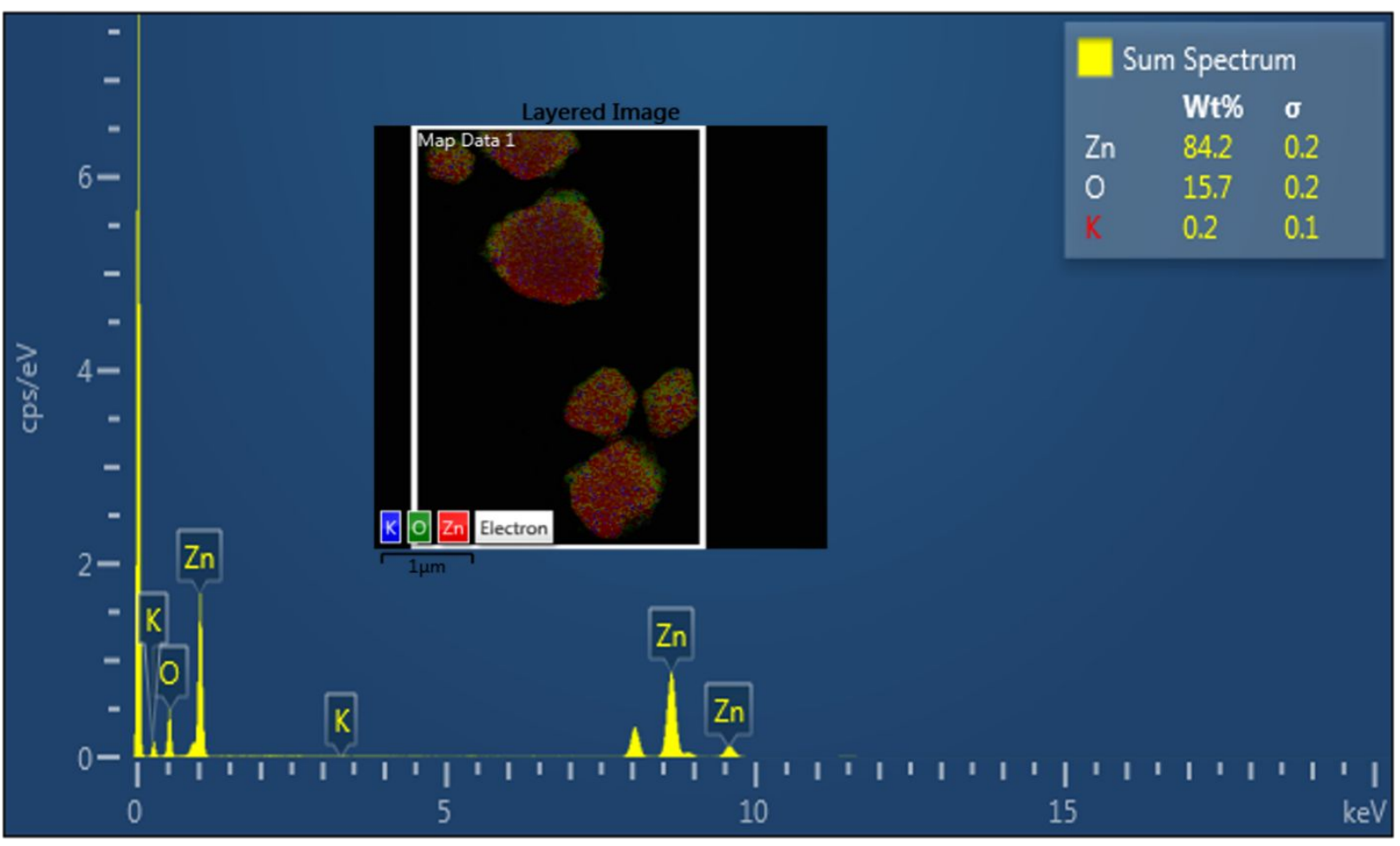

\begin{tabular}{|l|c|r|l|r|r|r|r|}
\hline Element & $\begin{array}{c}\text { Line } \\
\text { Type }\end{array}$ & k Factor & k Factor type & $\begin{array}{c}\text { Absorption } \\
\text { Correction }\end{array}$ & Wt\% & \multicolumn{1}{|c|}{$\begin{array}{c}\text { Wt\% } \\
\text { Sigma }\end{array}$} & Atomic \% \\
\hline $\mathrm{O}$ & K series & 1.455 & Theoretical & 1.00 & 15.66 & 0.24 & 43.11 \\
\hline $\mathrm{K}$ & K series & 0.990 & Theoretical & 1.00 & 0.18 & 0.05 & 0.20 \\
\hline $\mathrm{Zn}$ & K series & 1.496 & Theoretical & 1.00 & 84.16 & 0.25 & 56.69 \\
\hline Total: & & & & & 100.00 & & 100.00 \\
\hline
\end{tabular}


S3. Hall effect measurements were carried out by four probe method in Vander Pauw configuration. The carrier concentration was calculated using equation: $V_{H}=-\left(\frac{1}{n q}\right) \frac{I_{x} B_{z}}{t}$, where $I_{x}$ is constant current flowing through the pellet and $B_{z}$ is magnetic field strength perpendicular to current direction, $\mathrm{t}$ is thickness of pellet and $\mathrm{n}$ is carrier concentration per unit volume, $\mathrm{q}$ is electric charge. The Hall coefficient $\left(\mathrm{R}_{\mathrm{H}}=1 / \mathrm{nq}\right)$ and carrier concentration (n) of pure and alkali doped $\mathrm{ZnO}$ samples is represented in Fig. S2.
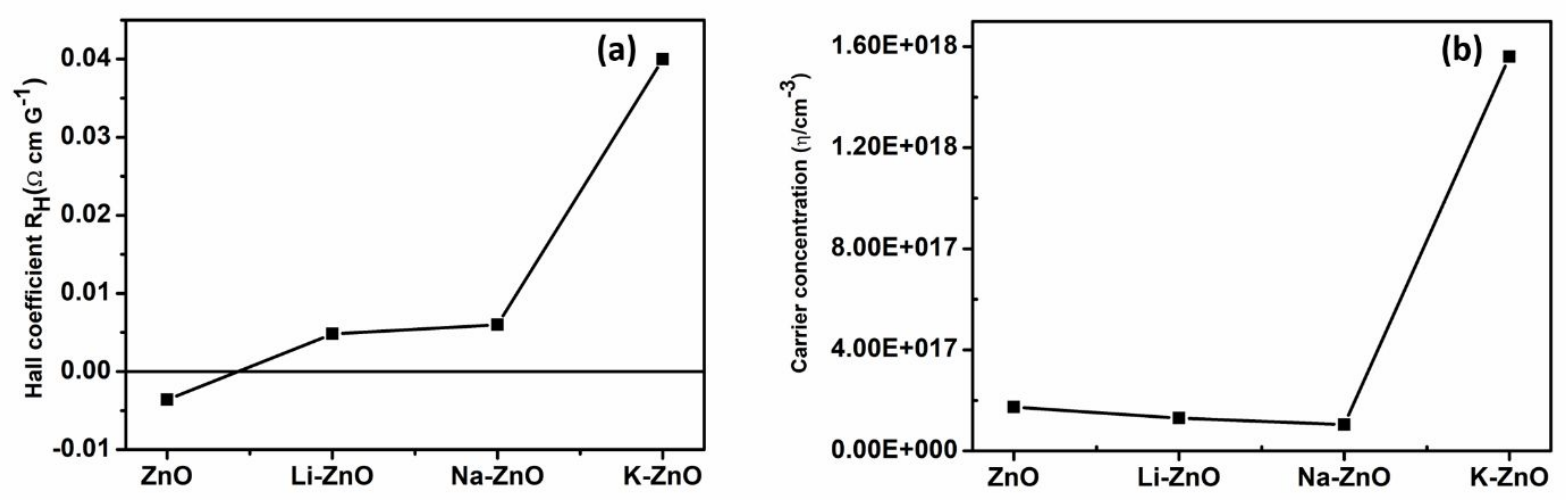

Fig.S3 Hall plots representing (a) Hall coefficient and type of conductivity (b) carrier concentration in alkali doped $\mathrm{ZnO}$ hydroelectric cell samples.

S4. Comparison of dielectric loss spectra of deionized (DI) water to alkali doped $\mathrm{ZnO}$ Hydroelectric cells dipped in DI water. The relaxation time for free water dipoles has been obtained at $\tau \sim 10^{-5} \mathrm{~s}$. A clear shift of dielectric relaxation peaks towards higher frequency is visible in alkali doped $\mathrm{ZnO}$ samples as compared to loss peak of pure water. The calculated relaxation time for reorientational polarization for alkali doped wet Hydroelectric are: $\tau \sim 2.41 \times 10^{-7} \mathrm{~s}$ for $\mathrm{Li}-\mathrm{ZnO}$, $\tau \sim 2.56 \times 10^{-6} \mathrm{~s}$ for $\mathrm{Na}-\mathrm{ZnO}$ and $\tau \sim 5.30 \times 10^{-7} \mathrm{~s}$ for $\mathrm{K}-\mathrm{ZnO}$. 

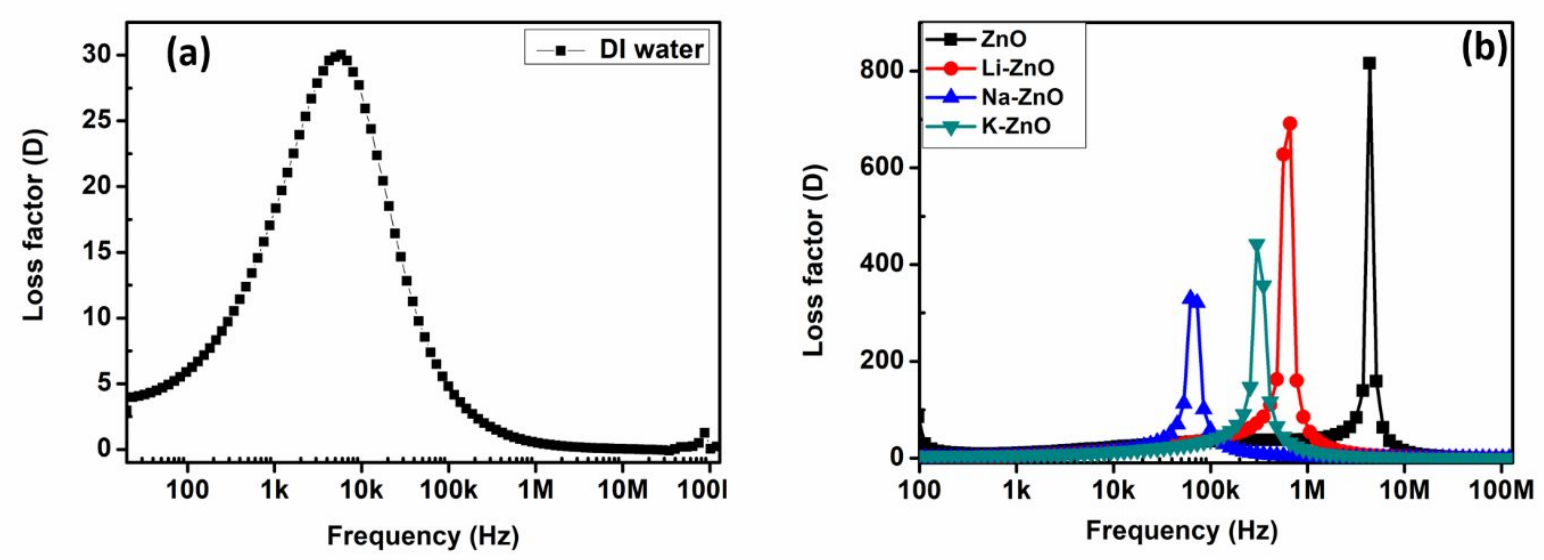

Fig.S4 (a) Dielectric loss peak of DI water representing dipolar relaxation (b) shift of relation peak towards higher frequency in pure and alkali doped $\mathrm{ZnO}$ samples.

S5. Nyquist plots for Dry ZnO based Hydroelectric cells where a single semicircular arc of very high resistance can be seen. It represents very high ohmic as well as charge transfer resistance of the order of $\sim 10^{7} \Omega$ for dry $\mathrm{ZnO}$ based Hydroelectric cells.

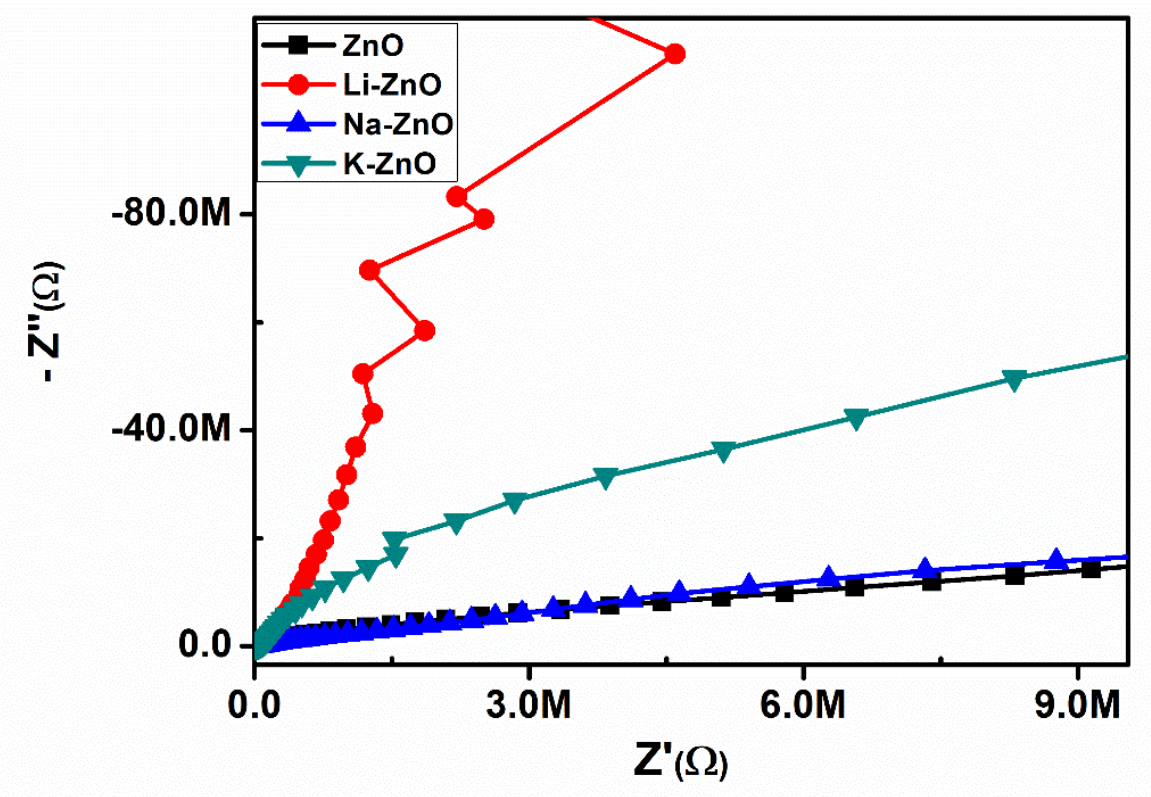


Fig. S5 Comparison of Nyquist plots for Dry ZnO based Hydroelectric cells representing highly resistive ohmic and charge transfer arcs.

S6 A representation of equivalent circuit used for modeling the experimental data of Nyquist plots of wet alkali doped $\mathrm{ZnO}$ Hydroelectric cells.

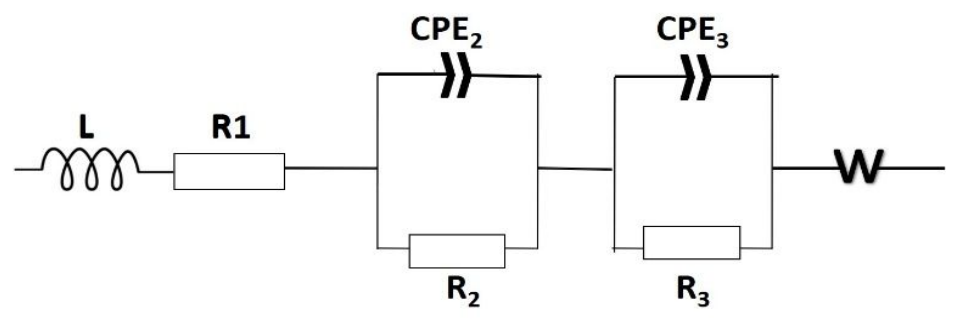

Fig. S6 Equivalent circuit used for theoretical fitting the experimental Nyquist plots of pure and alkali doped $\mathrm{ZnO}$ hydroelectric cells.

The equivalent circuit comprises of a Warburg element, two Zarc elements $\left(\mathrm{R}_{2} \| C P E_{2}\right) \&$ $\left(\mathrm{R}_{3} \| \mathrm{CPE}_{3}\right)$ and an inductor $(\mathrm{L})$ connected in series with a resistor $\left(\mathrm{R}_{1}\right)$. Inductive reactance of metallic elements in cell was represented by $L$ whereas $R_{i}$ represents internal ohmic resistance of active material in the cell, $\mathrm{R}_{1}$ and $\mathrm{CPE}_{1}$ represents ion/solid interface reactance \& capacitance formed during cycling at anode surface, $\mathrm{R}_{2}$ and $\mathrm{CPE}_{2}$ represents charge transfer reactance \& capacitance at anode surface and $\mathrm{W}$ represents diffusion in active material of electrodes at very low frequency.

Table S1: Fitting parameters of temperature dependent dielectric relaxation plots by Macedo \& Litovitz model.

\begin{tabular}{|l|l|l|l|l|}
\hline Sample & $E_{a}(K J / m o l)$ & $E_{b}(K J / m o l)$ & $\tau_{0}(\mathbf{s})$ & C \\
\hline Pure ZnO & 154.05 & 38.78 & $4.00598 \mathrm{E}-29$ & $1.62 \mathrm{E} 7$ \\
\hline Li-ZnO & 141.93 & 38.96 & $5.80699 \mathrm{E}-29$ & $7.31 \mathrm{E} 6$ \\
\hline
\end{tabular}




\begin{tabular}{|l|l|l|l|l|}
\hline Na-ZnO & 145.82 & 28.41 & $2.25219 \mathrm{E}-29$ & $0.17 \mathrm{E} 6$ \\
\hline K-ZnO & 128.54 & 33.13 & $1.04664 \mathrm{E}-27$ & $1.06 \mathrm{E} 6$ \\
\hline
\end{tabular}

Table S2: Equivalent Circuit Fitting Parameters of Nyquist data of wet $\mathrm{ZnO}$ based Hydroelectric cell

\begin{tabular}{|l|l|l|l|l|l|l|l|l|l|l|}
\hline Sample & $\mathbf{R i}(\boldsymbol{\Omega})$ & $\mathbf{R}_{\mathbf{1}}(\boldsymbol{\Omega})$ & $\mathbf{R}_{\mathbf{2}}(\boldsymbol{\Omega})$ & $\mathrm{CPE}_{\mathbf{1}}(\mathbf{F})$ & $\mathbf{C P E}_{\mathbf{2}}(\mathbf{F})$ & $\mathbf{n}_{\mathbf{1}}$ & $\mathbf{n}_{\mathbf{2}}$ & $\mathbf{L}(\mathbf{H})$ & $\mathbf{W}_{\text {s1 }}$ & $\mathbf{W}_{\text {s2 }}$ \\
\hline Pure ZnO & 66.86 & 7.15 & 5.47 & $8.7 \mathrm{E}-5$ & $6.46 \mathrm{E}-5$ & 0.78 & 0.47 & $4.0 \mathrm{E}-8$ & 440 & 0.032 \\
\hline Li-ZnO & 27.41 & 4.42 & 5.32 & $3.6 \mathrm{E}-5$ & $2.5 \mathrm{E}-4$ & 0.64 & 0.69 & $4.5 \mathrm{E}-7$ & 74 & 7.4 \\
\hline Na-ZnO & 10.95 & 4.26 & 6.01 & $2.7 \mathrm{E}-4$ & $7.46 \mathrm{E}-4$ & 0.78 & 0.59 & $7.5 \mathrm{E}-10$ & 107 & 5.5 \\
\hline K-ZnO & 3.60 & 13.69 & 4.05 & $4.9 \mathrm{E}-4$ & $8.04 \mathrm{E}-5$ & 0.94 & 1 & $7.4 \mathrm{E}-8$ & 28 & 6.8 \\
\hline
\end{tabular}

